\title{
The Contribution of Sucrose Metabolism Enzymes to Sucrose Accumulation in Cucumis melo
}

\author{
Yosef Burger $^{1}$ \\ Newe-Ya'ar Research Center, Agricultural Research Organization, P.O. Box 1021, \\ Ramat Yishay, 30009, Israel \\ Arthur A. Schaffer \\ Department of Vegetable Research, Volcani Center, Agricultural Research Organization, \\ Bet Dagan, 50250, Israel
}

\begin{abstract}
Additional INDEX words. melon, acid invertase, neutral invertase, sucrose synthase, sucrose phosphate synthase
Abstract. The relationship between sugar accumulation and sucrose metabolism enzyme activities was studied among seven genotypes of Cucumis melo $\mathrm{L}$., covering the broad genetic range of sucrose accumulation found in the species. The primary determinant correlated with sucrose levels was the genetic variation for developmental loss of soluble acid invertase (AI) activity. Sucrose accumulation in the developing fruit began only when AI activity declined to less than an experimentally determined threshold value, and continued until removal of the fruit from the plant. In addition, the activities of sucrose phosphate synthase (SPS), sucrose synthase (SuSy), and neutral invertase (NI) were all positively correlated with sucrose accumulation among the genotypes. The low-sucrose-accumulating genotypes were characterized by low activities of each of the three enzymes, irrespective of their invertase activities. Final sucrose content was best predicted for each genotype by the number of days the fruit remained attached to the plant while characterized by "sucrose accumulation metabolism," which was characterized primarily by AI activity less than threshold values, together with SPS, SuSy, and NI activities higher than threshold levels.
\end{abstract}

Fruit quality of melon is determined primarily by its sugar content (Yamaguchi et al., 1977). The mature fruit sugar content in sweet melons is comprised of the disaccharide sucrose and its two hydrolysis products, the hexoses glucose and fructose. However, the increase in total sugar content during fruit maturation is due particularly to the accumulation of sucrose during the final stages of fruit development (Burger et al., 2000; Hubbard et al., 1989, 1991; Lingle and Dunlap, 1987; McCollum et al., 1988; Rosa, 1928; Schaffer et al., 1987, 1996). Variation in sucrose levels also accounts for the genetic differences in total sugar contents (Burger et al., 2000; Stepansky et al., 1999) and for the natural variability within a particular cultivar as a result of environmental and developmental differences (Burger et al., 2000).

Although source primary photosynthate production will play a role in determining the availability of assimilate supply, the accumulation of sucrose appears to be controlled by the metabolism of carbohydrates in the fruit sink itself (Hubbard et al., 1989; Lester et al., 2001, Schaffer et al., 1996, 2000). Sucrose and the galactosyl-sucrose oligosaccharides, raffinose and stachyose, are translocated from the source to fruit sink in the Cucurbitaceae family, including Cucumis melo (Chrost and Schmitz, 1997; Mitchell et al., 1992). The near absence of raffinose and stachyose in the melon fruit flesh points to the rapid hydrolysis and metabolism of these translocated sugars in the fruit, or adjacent to it (Chrost and Schmitz, 1997; Hubbard et al., 1989; Hughes and Yamaguchi, 1983; Pharr and Hubbard, 1994).

Previous studies have shown that the melon fruit undergoes a metabolic transition from the stage of fruit growth to that of sucrose accumulation, characterized by a developmental loss

Received for publication 3 Oct. 2006. Accepted for publication 19 June 2007. We thank Mr. Shmuel Shen and Dr. Marina Petreikov for helpful assistance. ${ }^{1}$ Corresponding author. E-mail: burgery@agri.gov.il. of soluble acid invertase (AI) activity (Hubbard et al., 1989; Iwatsubo et al., 1992; Lester et al., 2001; McCollum, et al., 1988; Ranwala et al., 1991; Schaffer et al., 1987). The same phenomenon has been described for other sucrose-accumulating fruit (Hubbard et al., 1990, 1991; Schaffer et al., 1989), including species of tomato (Solanum lycopersicun L.) (Miron and Schaffer, 1991; Stommel, 1992; Yelle et al., 1991).

A key role for sucrose phosphate synthase (SPS) activity in sucrose-accumulating melon fruit was proposed by Hubbard et al. (1991), who showed that sucrose accumulation in melon fruit was characterized by a developmental increase in SPS activity, in addition to the developmental loss of AI activity. Lester et al. (2001) confirmed the importance of the loss in AI activity and the increase in SPS activity in two sweet melon cultivars and emphasized particularly the necessity for SPS activity to be higher than that of AI. An increase in SPS activity was similarly reported for other sucrose-accumulating fruit (Irving et al., 1997; Langenkamper et al., 1998; Miron and Schaffer, 1991; Yelle et al., 1991). The increase in SPS activity implies physiological significance and indicates the importance of sucrose synthesis in the fruit, perhaps as part of a hydrolysisresynthesis scheme of sucrose accumulation. As such, it was hypothesized to be a potential key controlling point in determining sugar accumulation and content (Miron and Schaffer, 1991). However, Klann et al. (1993) reported no significant differences in SPS activity between fruit of sucrose-accumulating and hexose-accumulating genotypes of tomatoes and, similarly, Stommel (1992) did not observe an increase in SPS activity concomitant with sucrose accumulation in the sucroseaccumulating wild tomato species Lycopersicon peruvianum (L.) Mill. (Solanum peruvianum L.).

Both sucrose synthase (Giaquinta, 1979; Moriguchi, et al., 1990, 1992; Schaffer et al., 1987; Suzuki et al., 1996) and neutral, or alkaline, invertase (Glasziou and Gayler, 1972; Kato 
and Kubota, 1978; Ricardo and Rees, 1970) activities have also been implicated in sucrose accumulation. However, the role of these enzymes in sucrose accumulation is not obvious because SuSy is generally associated with sucrose cleavage rather than synthesis, and neutral invertase (NI) catalyzes sucrose hydrolysis. Accordingly, Hubbard et al. (1989) related to SuSy and NI as cleavage enzymes, together with AI, in their calculations of the relationship between sucrose metabolism enzyme activities and sucrose accumulation. In the sugarcane (Saccharum officinarum L.) stem (Zhu et al., 1997), sucrose accumulation was associated with the developmental loss of AI activity and an increase in SPS activity; however, SuSy and NI activities were unrelated to the increase in sucrose content.

The putative roles of the aforementioned sucrose metabolizing enzymes have been hypothesized primarily from developmental correlative studies of a small number of genotypes, frequently only one or two in each study. Comparative and correlative studies based on small numbers of genotypes may be misleading. The purpose of the current research was to expand the study of the contributions of the sucrose metabolizing enzymes to sucrose accumulation, in light of the conflicting evidence for their role in sucrose-accumulating fruit. We took an approach that we feel minimizes the limits of correlative studies and compared, developmentally, a large number of genotypes that span the spectrum of genetic variability of sucrose accumulation.

The $C$. melo species comprises a spectrum of germplasm with a very broad range of sugar levels (Pitrat et al., 2000; Stepansky et al., 1999). At the one extreme are those primitive types with low sugar in the mature fruit, consisting primarily of only hexoses, glucose and fructose. At the other extreme are the cultivated high-sugar sweet dessert melons, characterized by high sucrose levels, in addition to the hexoses. Sugar levels in the species span the continuum from low to high, but the major component contributing to this continuum in total sugar concentration is specifically the sucrose levels (Burger et al., 2000; Stepansky et al., 1999). We took advantage of this genetic resource and compared sucrose accumulation and sucrose metabolism among $C$. melo genotypes ranging from low to high sucrose accumulation. In addition, we included also a single Cucumis sativus L. genotype, which does not accumulate sucrose.

\section{Materials and Methods}

Plant material. The following eight genotypes were used in this study, and are described in Table 1:1) C. melo cv. Galia (GAL), 2) C. melo breeding line Noy Yizre'el (NY), 3) C. melo breeding line Krimka (KRY), 4) C. melo breeding line Persian 202 [P202 (Paris et al., 1986)], 5) C. melo breeding line Persian 221 (P221), 6) C. melo cv. Black Skin (BSK), 7) C. melo breeding line Faqqous (FAQ), and 8) C. sativus cv. Delilah (DEL).

Table 1. Description of the genotypes of Cucumis L. used in the comparison of sucrose metabolism.

\begin{tabular}{lcll}
\hline Genotype & Abbreviation & \multicolumn{1}{c}{ Marketing type } & \multicolumn{1}{c}{ Group } \\
\hline Black Skin & BSK & Pickling & Conomon \\
Delilah & DEL & Cucumber, Bet Alfa & Sativus \\
Faqquos & FAQ & Pickling & Flexuosus \\
Galia & GAL & Galia & Reticulatus \\
Krimka & KRY & Galia & Reticulatus \\
Noy Yizre'el & NY & Haogen & Cantalupensis \\
Persia P202 & P202 & & Reticulatus \\
Persia P221 & P221 & & Reticulatus \\
\hline
\end{tabular}

Plants were grown in the open field in the Yizre'el Valley, Israel, as a summer crop under standard techniques. Flowers were tagged at anthesis, and a maximum of two fruit were allowed to develop per plant. Fruit were harvested throughout fruit development until abscission, including the low-sugar C. melo breeding line FAQ, and C. sativus cv. Delilah.

Sugar ANALYSES. Harvested fruit were brought to the laboratory and sampled as follows. About $1 \mathrm{~g}$ fresh weight mesocarp tissue, taken from the center-equatorial portion of the fruit, was placed in $80 \% \mathrm{EtOH}$ and stored at $-20{ }^{\circ} \mathrm{C}$ until extraction. Sugars were extracted and analyzed colorimetrically as sucrose and reducing sugars according to the methods of Sumner (1921) and Van Handel (1968), respectively, as previously described (Schaffer et al., 1987).

EnZYME DETERminations. Additional samples from the same fruit mesocarp were immediately frozen under liquid nitrogen and stored at $-78{ }^{\circ} \mathrm{C}$ for determination of enzyme activities. Soluble AI and NI activities were assayed as reported in Schaffer et al. (1987). Insoluble AI was not measured in this study because our initial study (Schaffer et al., 1987) indicated that this activity does not developmentally change with regard to sucrose accumulation. Because neutral NI is frequently masked by residual activity of $\mathrm{AI}$ at a $\mathrm{pH}$ of 7 , we estimated individual invertase activities using the ratio of activity at a $\mathrm{pH}$ of 5 and 7 for each of the two activities (Kato and Kubota, 1978). The AI and NI enzymes were separated by differential ammonium sulfate precipitation [AI precipitated at $60 \%$ to $80 \%$, NI precipitated at 20\% to $40 \%$ (Chen and Black, 1992; Ricardo and Rees, 1970)]. The pH activity curve of each enzyme was calculated and the following equation was used to determine the individual activities: $\mathrm{AI}=$ activity at $\mathrm{pH} 5.0-(0.25 \times$ activity at $\mathrm{pH} 7.0) ; \mathrm{NI}=$ activity at $\mathrm{pH} 7.0-(0.30 \times$ activity at $\mathrm{pH} 5.0)$.

Sucrose phosphate synthase activity was assayed in the synthesis direction as in Miron and Schaffer (1991). One gram of frozen tissue kept in liquid nitrogen was ground in a cold mortar in $3 \mathrm{~mL}$ buffer containing $50 \mathrm{~mm}$ Hepes- $\mathrm{NaOH}(\mathrm{pH}$, 7.5), $5 \mathrm{~mm} \mathrm{MgCl}_{2}, 1 \mathrm{~mm} \mathrm{Na}-E D T A, 2.5 \mathrm{~mm}$ DTT, $0.5 \mathrm{mg} \cdot \mathrm{mL}^{-1}$ Bovine serum albumin, and 0.05\% (v/v) Triton X-100 (Sigma, St. Louis) according to the method of Hubbard et al. (1989), with slight modifications. A 1-mL portion of the homogenate was desalted directly by centrifugal filtration (Helmerhorst and Stokes, 1980) at $1{ }^{\circ} \mathrm{C}$ on 5-mL Sephadex G-25 columns (Sigma), equilibrated with the buffer mentioned earlier but without EDTA and Triton X-100. Sucrose phosphate synthase was determined in reaction mixtures $(70 \mu \mathrm{L})$ containing $50 \mathrm{~mm}$ Hepes- $\mathrm{NaOH}$ (pH, 7.5), $15 \mathrm{~mm} \mathrm{MgCl}_{2}, 25 \mathrm{~mm}$ Fru6P, $25 \mathrm{~mm}$ Glc6P, $25 \mathrm{~mm}$ UDPGlc, and $40 \mu \mathrm{L}$ extract. Mixtures were incubated for $30 \mathrm{~min}$ at $37^{\circ} \mathrm{C}$, and incubation was ended with the addition of $70 \mu \mathrm{L} 30 \% \mathrm{KOH}$. Enzyme blanks were ended with $\mathrm{KOH}$ at $0 \mathrm{~min}$. Sucrose was assayed according to the modified anthrone method of Van Handel (1968). Sucrose synthase was assayed as noted earlier but with $25 \mathrm{~mm}$ Fru instead of Fru6P, and in the absence of Glc6P.

Statistical anAlysis. Statistical analysis and stepwise regression analysis were performed by JMP (version 5.1; SAS Institute, Cary, NC).

\section{Results}

The genotypes studied represent a continuum of sucrose accumulation, as seen in Fig. 1A. The sweet melon, high-sugar genotypes NY, KRY, and GAL are characterized by the highest 

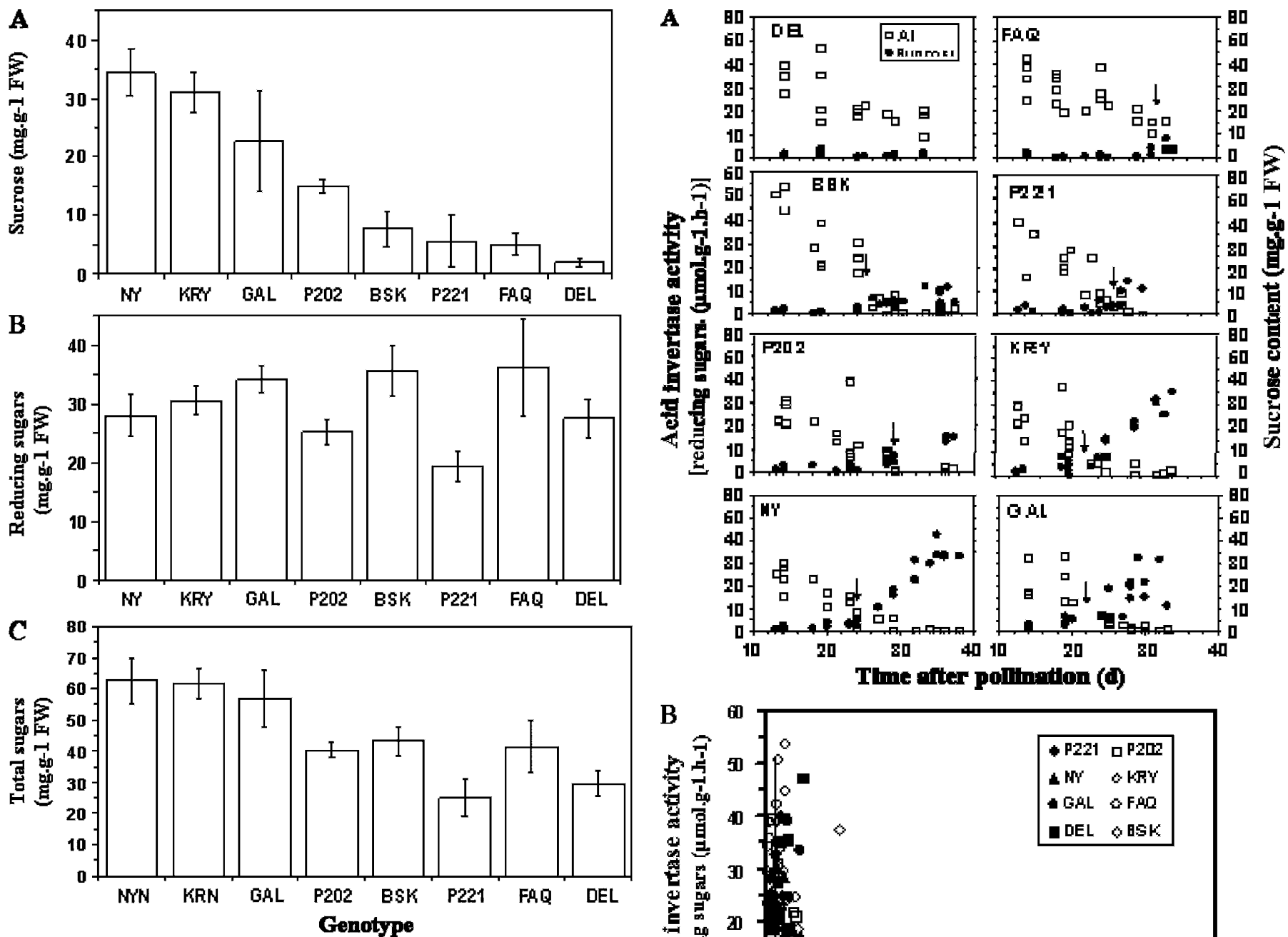

Fig. 1. (A-C) Sucrose (A), reducing sugars (B), and total sugars (C) of mature fruit of eight Cucumis genotypes: Noy Yizre'el (NY), Krymka (KRY), Galia (GAL), Persia 202 (P202), 'Black Skin' (BSK), Persia 221 (P221), Faqqous (FAQ), and 'Delilah' (DEL). Averages are of a minimum of five mature fruit of each genotype. Data are presented on a fresh weight basis.

sucrose levels. The low-sugar genotypes have respectively lower sucrose levels. Although there is variation in levels of reducing sugars between the genotypes, the contribution to the differences in total sugar content is small (Fig. 1B, C).

The developmental pattern of sucrose accumulation in the eight genotypes studied, together with the parallel developmental AI enzyme activity pattern for each of the genotypes, is shown in Fig. 2A. All the genotypes studied were characterized by a developmental reduction in AI activity, and in no genotype did AI activity increase during development. The most striking observation is that across the eight genotypes, sucrose accumulation began only at the stage at which AI activity declined below a threshold value (designated by the arrow in Fig. 2A).

The threshold value for AI below which sucrose accumulation occurs could be experimentally calculated. Figure 2B shows the composite data of AI activity and sucrose contents of fruit of all eight genotypes and all developmental stages. With the assay conditions used in this study, the threshold value was determined to be $\approx 7 \mu \mathrm{mol}$ reducing sugars/gram fresh weight/hour. In the case of the C. sativus genotype DEL, which does not accumulate sucrose, AI activity remained above this

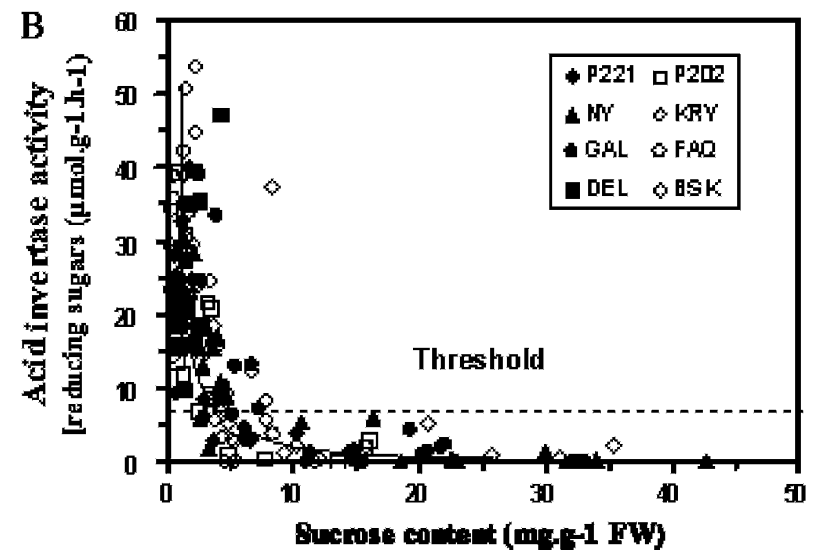

Fig. 2. (A, B) Acid invertase activity and sucrose content of developing fruit mesocarp of eight genotypes of Cucumis. Acid invertase activity (A) and sucrose content during fruit development (B). Relationship between acid invertase activity and sucrose content of all the fruit presented in (A). Regression curve line was calculated as $\mathrm{y}=37.21 \mathrm{x}^{-1.2}, r=0.72$. The arrow in each graph represents the stage that acid invertase activity declines below threshold and sucrose begins to accumulate. The eight genotypes are as follows: Noy Yizre'el (NY), Krymka (KRY), Galia (GAL), Persia 202 (P202), 'Black Skin' (BSK), Persia 221 (P221), Faqqous (FAQ), and 'Delilah' (DEL). Data are presented on a fresh weight basis. Each point represents data from a single fruit from a single plant.

threshold value until abscission and approached the threshold value in the most mature fruit (Fig. 2A). The AI activity of the C. melo FAQ genotype declined to below threshold values only in the most mature fruit, at the stage of complete abscission, and subsequently had only a minimal period of sucrose accumulation. The potential sucrose accumulation days can be calculated for each genotype according to the decline of AI activity below the threshold value until the fruit is ripe and either abscises or is removed.

Developmental patterns of SPS, SuSy, and NI activities for each of the genotypes are presented in Fig. 3A. Developmental 

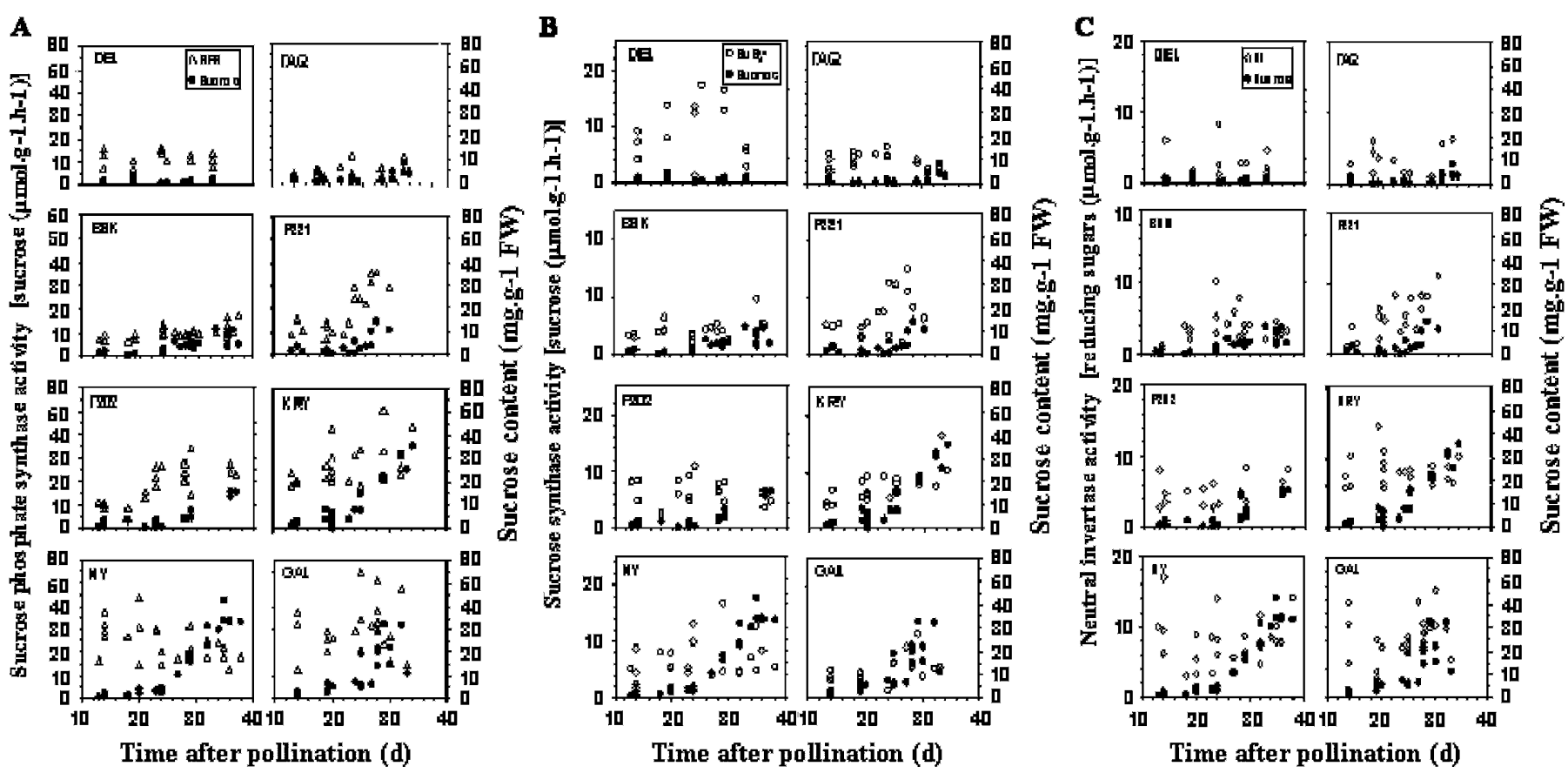

Fig. 3. (A-C) Enzyme activities of (A) sucrose phosphate synthase (SPS), (B) sucrose synthase (SuSy), and (C) neutral invertase (NI), and sucrose content of developing fruit mesocarp of eight genotypes of Cucumis: Noy Yizre'el (NY), Krymka (KRY), Galia (GAL), Persia 202 (P202), 'Black Skin' (BSK), Persia 221 (P221), Faqqous (FAQ), and 'Delilah' (DEL). Data are presented on a fresh weight basis. Each point represents data from a single fruit from a single plant.

increases in activities were not consistently observed in the comparison of the large number of genotypes for any of the enzymes, and the clear pattern observed for AI is not consistently observed for these three enzymes. The nonaccumulating genotypes FAQ and BSK show low activities of each of the three enzymes throughout development, whereas DEL shows low activities of SPS and NI. The remaining sucrose accumulators show higher SPS activities, but their developmental patterns differ. Although P202, P221, GAL, and KRY show developmental increases in activity coinciding with the increases in sucrose levels, NY exhibits a high activity already in the young fruit, before the onset of sucrose accumulation, and a subsequent modest decline in activity. A similar pattern of SPS activity during NY development was observed in a separate year's harvest (data not presented). Significantly, the activities of SPS during the sucrose accumulation phase of P202 and P221 are not significantly lower than those of NY during its sucrose accumulation phase.

When all the data for SPS activity of the eight genotypes throughout development are plotted against fruit sucrose content (Fig. 4A), it appears that the contribution of SPS activity to sucrose accumulation follows a saturation pattern in which significant SPS activity $(\approx 15 \mu \mathrm{mol}$ sucrose synthesized/gram fresh weight/hour in our saturating assay) is present in fruit accumulating significant levels of sucrose. Once above this level, there does not appear to be an additive contribution of SPS activity to sucrose content. On the other hand, the lowsucrose-accumulating BSK, characterized by a large potential sucrose accumulation period based on AI activity, has low SPS activity throughout fruit development.

Similar results are observed for SuSy (Figs. 3B, 4B) and NI (Figs. 3C, 4C). Similar to SPS, both high- and moderatesucrose-accumulating fruit are characterized by higher activities $(\approx 5 \mu \mathrm{mol}$ sucrose/gram fresh weight/hour) of both SuSy and NI, whereas the low-sucrose-accumulating genotypes may be characterized by either the low or high activities. Also, similar to SPS, there is no additive contribution of activities above this value to sucrose accumulation. The genotypes FAQ and BSK have low SuSy and NI activities throughout development. 'Delilah' has a high SuSy activity, particularly during the earlier stages of fruit development, together with its high AI activity.

The relative contribution of each of the four enzymes to sucrose accumulation was calculated by a stepwise regression analysis. The days of low AI activity was calculated as the number of days of each particular fruit following the decline to below-threshold activity, based on the data presented in Fig. $2 \mathrm{~A}$. The additional enzymes were expressed as their activity in the particular fruit analyzed. The results presented in the stepwise partial $r^{2}$ column of Table 2 show strikingly that the overwhelming component accounting for differences in sucrose levels in the melon germplasm is the number of days that the fruit remains attached to the vine after the soluble AI activity declines to below-threshold values.

Figure 5A presents the relationship between the sucrose content of mature fruit of the eight genotypes and the number of days from the decline in AI activity to below-threshold values to maturity for each of the genotypes, as determined from Fig. 1. The nonaccumulating and low-sucrose genotypes (DEL, FAQ, and P221) show from none to only a few days of low AI activity before abscission and, hence, low potential sucrose accumulation. The high-sucrose accumulators are characterized by a longer period of fruit development with low AI activity. These data point to the number of potential sucrose accumulation days, resulting from low AI activity, as the key factor in the genetic determination of sucrose accumulation. However, this single factor alone does not completely account for all the genetic variation in sucrose accumulation of the 


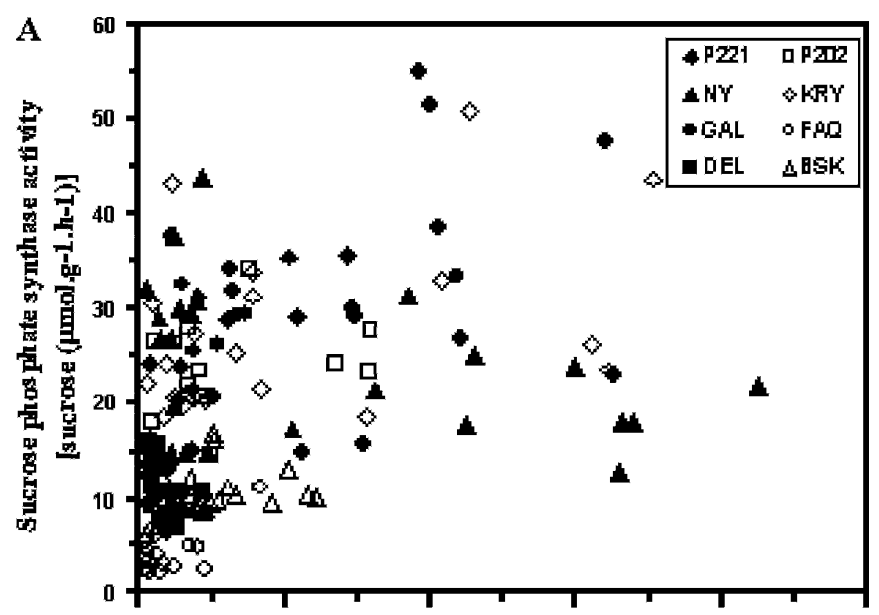

B
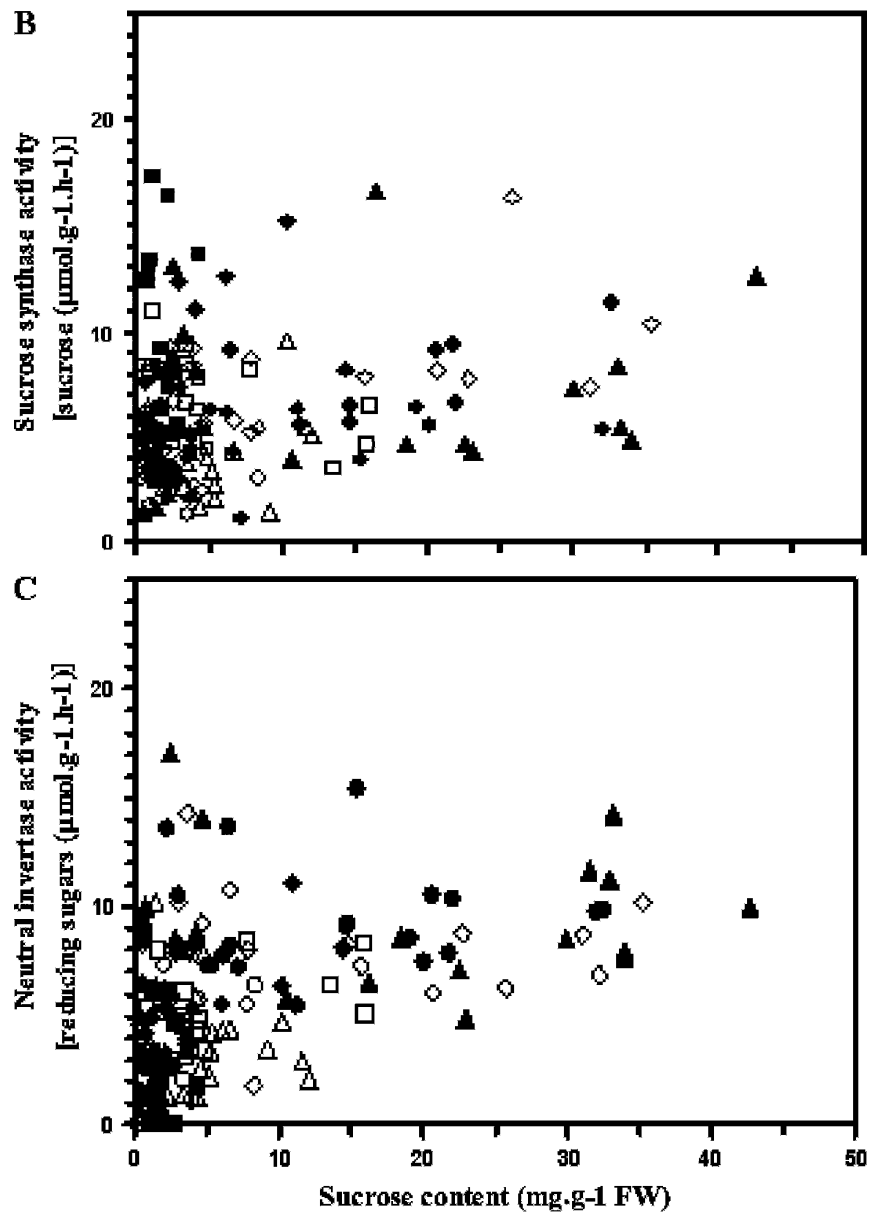

Fig. 4. Relationship between activities of (A) sucrose phosphate synthase (SPS), (B) sucrose synthase (SuSy), and (C) neutral invertase (NI), and sucrose content of all the fruit from eight genotypes of Cucumis: Noy Yizre'el (NY), Krymka (KRY), Galia (GAL), Persia 202 (P202), 'Black Skin' (BSK), Persia 221 (P221), Faqqous (FAQ), and 'Delilah' (DEL). Data are presented on a fresh weight basis. Each point represents data from a single fruit from a single plant. Regression curve lines for the three enzymes are as follows: SPS, $\mathrm{y}=10.21 \mathrm{x}^{0.31}, r=0.52 ; \operatorname{SuSy}, \mathrm{y}=0.5 \operatorname{Ln}(\mathrm{x})+5.6, r=0.18 ; \mathrm{NI}, \mathrm{y}=3.029 \mathrm{x}^{0.29}$, $r=0.42$.

broad spectrum of genotypes studied. 'Black Skin', for example, has a high number of potential sucrose accumulation days according to AI activity, but, nevertheless, has a low-sucrose level at maturity. Furthermore, the negative curvilinear response in Fig. 1A also indicates that although AI activity
Table 2. Stepwise regression analysis for sucrose levels in mature Cucumis fruit.

\begin{tabular}{lccc}
\hline Component & $R^{2}$ & Stepwise partial $R^{2}$ & $P$ \\
\hline Days with low AI & 0.434 & 0.4344 & 0.0000 \\
NI & 0.709 & 0.2669 & 0.0000 \\
SuSy & 0.739 & 0.0374 & 0.0233 \\
SPS & 0.739 & 0.0002 & 0.8762 \\
Days after pollination & 0.746 & 0.0074 & 0.3036
\end{tabular}

AI, acid invertase; NI, neutral invertase; SPS, sucrose phosphate synthase; SuSy, sucrose synthase.
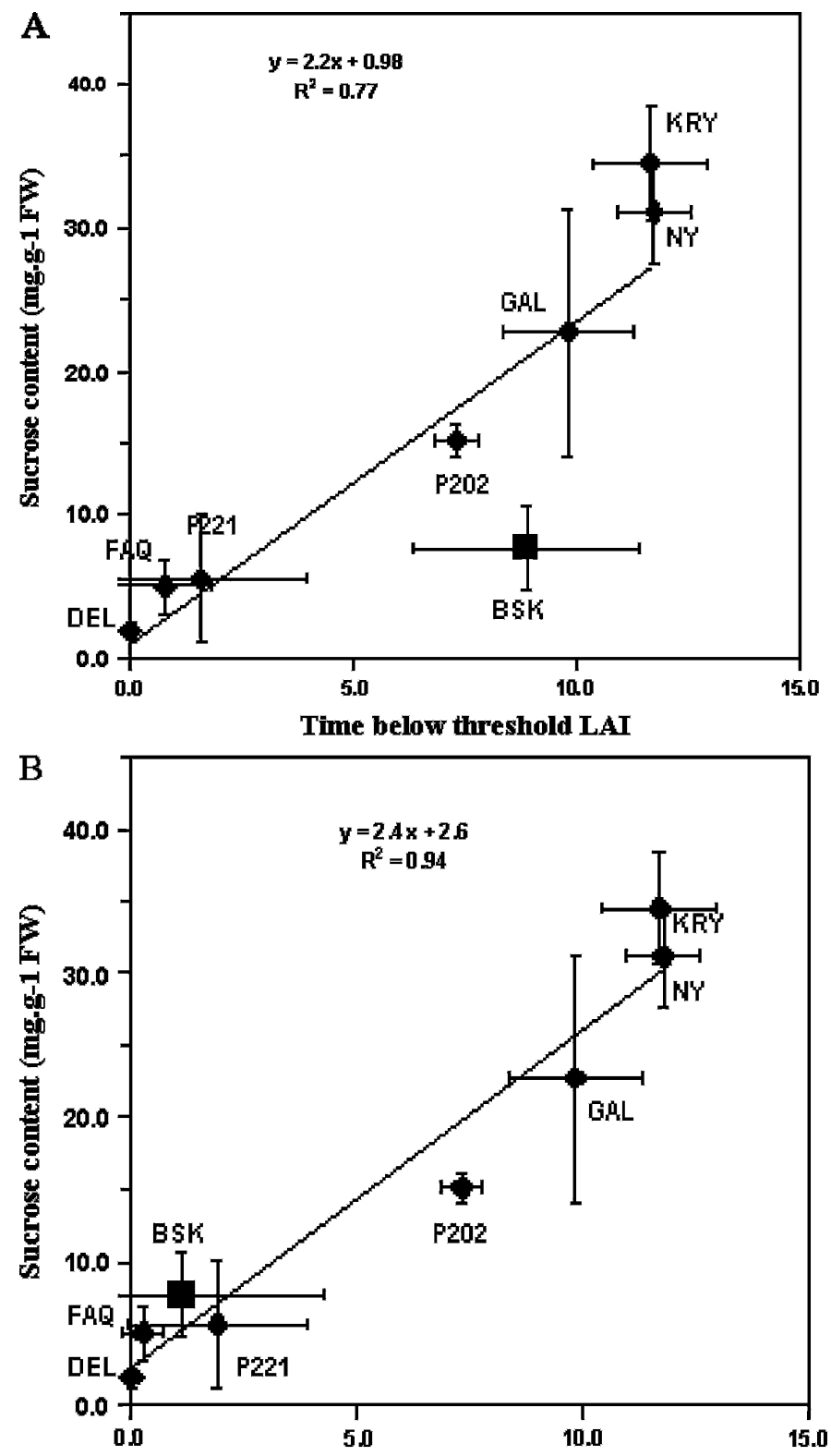

Time below threshold LAI + SAM

Fig. 5. (A, B) Correlation between the mature fruit sucrose content and (A) number of days with below-threshold acid invertase activity (LAI) and (B) number of days with sucrose accumulation metabolism (SAM) among eight genotypes of Cucumis: Noy Yizre'el (NY), Krymka (KRY), Galia (GAL), Persia 202 (P202), 'Black Skin' (BSK), Persia 221 (P221), Faqqous (FAQ), and 'Delilah' (DEL). Data are presented on a fresh weight basis. Each point represents data from a single fruit from a single plant. 
may be low, it is not necessarily accompanied by sucrose accumulation. Figure 5B improves upon the correlation of sucrose content and days of accumulation by presenting the results of our study in terms of the correlation of final sucrose content of each cultivar with the number of days of sucrose accumulation metabolism, representing the number of days characterized by both of the following components: 1) lower than threshold AI activity, together with 2) higher than threshold activities of the three additional enzymes positively correlated with sucrose accumulation.

\section{Discussion}

Perhaps the most significant factor that determines sucrose accumulation in melon fruit is the fate of the imported assimilate upon reaching the fruit sink. Although the metabolic fate of the translocated raffinose oligosaccharides stachyose and raffinose is their immediate hydrolysis, the fate of the translocated sucrose moieties can be either hydrolysis or storage as sucrose (Schaffer et al., 1996). Sucrose accumulation metabolism is characterized predominantly by low AI activity, which allows for sucrose maintenance and accumulation (Quick and Schaffer, 1996).

The onset of the developmental phase of sucrose accumulation in melon fruit is concomitant with the decline in AI activity to a below-threshold level. Analogous results have been obtained in a comparison of sugarcane genotypes (Zhu et al., 1997) as well as for a broad phylogenetic spectrum of leaves undergoing the sink-to-source transition (Goldschmidt and Huber, 1992). However, in addition to the requirement for low AI activity, our results clearly indicate that sucrose accumulation occurs only in the presence of threshold activities of the other three enzymes directly involved in sucrose metabolism.

One of the most significant contributions of our multigenotype study is that not only SPS but also SuSy and NI activities are positively, or at least not negatively, correlated with sucrose accumulation. Although a contributory role for SPS has generally been posited in sucrose accumulation (Hubbard et al., 1989, 1991; Lester et al., 2001; Zhu et al., 1997), the roles of other two enzymes, SuSy and NI, have been less clear. Some studies have attributed sucrose breakdown roles to these two latter enzymes (Hubbard et al., 1989), but our study clearly indicates that increased activities of SuSy and NI characterize sucrose accumulation in $C$. melo, rather than the opposite. Sucrose synthase has previously been suggested to contribute positively to sucrose accumulation in melon (Schaffer et al., 1987), as well as other fruit (Moriguchi et al., 1990, 1992). A contributory role for SuSy can be easily explained because the enzyme catalyzes a reversible reaction and the direction of cytosolic sucrose synthesis is compatible with low-sucrose concentrations in the cytosol (Avigad, 1982). However, during the early stages of fruit development, particularly in the DEL genotype, SuSy may likely play a role in sucrose catabolism.

Neutral invertase has also been associated with sucrose storage in some of the early literature dealing with sucrose accumulation in plants (Hatch and Glasziou, 1963; Ricardo and Rees, 1970; Sacher et al., 1963). However, its role has generally been associated with sucrose breakdown and the decrease in sucrose accumulation (Hubbard et al., 1991; Ranwala et al., 1991). In fact, the physiological role of NI in sucrose metabolism is not clear, and the positive correlation with sucrose accumulation in our study suggests the need to rethink this step of sucrose metabolism in the context of sink sugar accumulation. It may play a necessary role in sucrose cycling in sucrosestoring tissues lacking AI activity and, as such, is not causal to, but rather indicative of, sucrose accumulation resulting from the absence of the alternative AI for necessary sucrose hydrolysis.

A point that should be made in this context is that frequently assays of NI reported in the literature are carried out in the absence of proper controls that distinguish between actual NI activity and residual AI activity, which takes place at a neutral $\mathrm{pH}$. The AI enzyme will hydrolyze significant amounts of sucrose at a neutral $\mathrm{pH}$, so that the distinctive contributions of the two enzymes to sucrose hydrolysis needs to be calculated. This can be done by chromatographically separating the two enzymes (Gao et al., 1999), but this method does not lend itself to multiple sample comparisons. Alternatively, the $\mathrm{pH}$ activity curve of either a purified AI extract or an AI extract with insignificant NI activity can be determined, and the calculation of the two invertase activities can be performed taking into consideration residual activities. The results presented in this study are based on this latter method, as described in the Methods and Materials section.

After the onset of sucrose accumulation, characterized by below-threshold AI activity and above-threshold SPS, SuSy, and NI activities, the continued accumulation of sucrose appears to be a developmental linear process. Accordingly, the final sucrose content of the harvested fruit will depend on the length of time that the fruit remains on the photosynthesizing vine, taking into consideration the source-sink interactions at the whole plant level, which will also affect the amount of photoassimilate reaching the fruit sink. The breeding line P221, which shows the metabolic pattern of sucrose accumulation (low AI and high SPS, NI, and SuSy), nevertheless has a low final sucrose content, emphasizing the importance of the length of period of sucrose accumulation metabolism for the accumulation of sucrose, as its sucrose accumulation period is relatively short.

The contribution of SPS, SuSy, and NI enzymatic activities, in addition to the developmental loss of AI activity, to sucrose accumulation is particularly striking in the low-sucrose, pickling melon genotype BSK. Its period of potential sucrose accumulation, as determined by days of low AI activity alone, is characteristic of genotypes with higher sucrose contents; however, it is also characterized by low activities of each of the other three sucrose metabolism enzymes during the potential sucrose accumulation period. Unfortunately, we did not observe a genotype among those studied with high activity of only one or two of the additional sucrose metabolism enzymes, which would have been useful in allowing us to determine whether, in fact, all three enzymes function as part of a sucrose accumulation metabolism complex, in conjunction with the low AI activity. However, the results suggest that all three enzymes behave in a coordinated manner, contributing to the sucrose accumulation phenomenon.

Considering the importance of the length of period of potential sucrose accumulation in determining final sugar content, the extension of this period is a warranted strategy for improving fruit quality. This may be accomplished via at least two approaches. The first is to control developmentally the loss of AI activity by genetic means, using either the available natural genetic variability in the $C$. melo species for timing of 
loss of activity or by molecular control of the timing of loss of activity. The latter will require additional study of the molecular control of the loss of AI activity in melon. Results by Iwatsubo et al. (1992) and Lester et al. (2001) indicate that the loss of AI activity in melon fruit is attributed to the developmental loss of the AI protein, and our results (Schaffer et al., 1987) indicate that the loss of AI activity is unrelated to proteinaceous invertase inhibitors, together suggesting that the molecular control of the loss of AI activity is likely at the transcriptional stage. The molecular control of AI activity in sucrose-accumulating tomato genotypes is similarly at the transcriptional stage (Klann et al., 1996; Miron et al., 2001).

The second strategy is to extend the length of the period from the loss of AI until harvest by delaying the ripening process, either genetically or by agrotechnical means. Welles and Buitlaar (1988) presented evidence that agrotechnical treatments (i.e., low night temperature) can increase fruit sugar content, in correlation with the increase in length of the fruit development period. We have similarly shown that reducing greenhouse nighttime temperatures from $18^{\circ} \mathrm{C}$ to $12^{\circ} \mathrm{C}$ during the period beginning with the onset of the sucrose accumulation phase led to a significant delay in fruit ripening, and the ripe fruit had a parallel increase in fruit sugar content (Burger et al., 2000). Conversely, high temperatures during the final stages of ripening may accelerate fruit ripening and increase respiratory loss of stored sugars, leading to lower sugar levels in the ripe fruit.

The absolute enzyme activities of SPS, SuSy, and NI during the respective accumulation periods do not appear to determine sucrose accumulation but, rather, there exists a threshold effect. The results presented in Figs. 3 and 4 indicate that although enzyme activities above the threshold value characterize the sucrose-accumulating fruit among the various genotypes, there does not appear to be any indication of a positive correlation between levels of sucrose and further increases in enzyme activities. If this conclusion is correct and, in fact, further increases in activities of these enzymes are not limiting factors to sucrose accumulation rates in sweet melon genotypes, then the further increase of these activities is not a warranted genetic breeding strategy for improving melon sugar content. The genetic overexpression of these enzyme activities in a fruitspecific, ripening-related manner would elegantly test this hypothesis. Although the sucrose-accumulating melon fruit have not yet been engineered to overexpress these enzymes, hexose-accumulating tomato fruit has been (Laporte et al., 1997; Nguyen-Quoc et al., 1999). Results indicate that sucrose unloading into the developing tomato fruit can be increased by overexpression of SPS in the developing fruit (Nguyen-Quoc et al., 1999), but it remains to be determined regarding to what extent these results can be extrapolated to the sucrose-accumulating melon fruit.

The SPS activities we observed in vitro under the saturating assay used are presumably not the in vivo physiological activities. Nevertheless, it may be significant to note that the experimentally determined threshold value for SPS activity associated with sucrose accumulation (Fig. 4A, >15 $\mu \mathrm{mol}$ sucrose/gram fresh weight/hour) is sufficient to account for $\approx 50$-fold of the observed sucrose accumulation rates $(0.3 \mu \mathrm{mol}$ sucrose/gram fresh weight/hour) in the sucrose-accumulating genotypes. The conversion of daily sucrose accumulation rates to hourly rates is based on assumptions such as continuous diurnal sucrose accumulation in the fruit sink, dependent on source photoassimilate reserves, because the melon fruit does not accumulate starch reserves, which can serve as a source for sucrose accumulation (Pratt, 1970; Rosa, 1928; Schaffer et al., 1987). Sucrose synthase activities were of an order of magnitude lower than those of SPS, a point emphasized by Hubbard et al. (1989) in stressing the role of SPS in sucrose accumulation. However, as indicated, our results show that not only SPS is associated with sucrose accumulation but SuSy is as well, and despite its lower activity compared with SPS, the in vitro SuSy activity alone can theoretically account for all the sucrose accumulation of the genotypes studied. However, as mentioned, the in vitro activities determined do not necessarily indicate the actual in vivo flux through the particular metabolic step, and conclusions cannot be made from the data obtained with regard to the actual in vivo flux pathway leading to sucrose accumulation.

Our previous study (Schaffer et al., 1987) used C. sativus (DEL) as an example of a nonaccumulating genotype to emphasize the importance of the developmental loss of AI activity to sucrose accumulation. The current study is the first report of a nonaccumulating $C$. melo genotype, FAQ, characterized by an extended maintenance of AI activity. This genetic material, as a result of its ready crossability with other genotypes of $C$. melo, allows for the study of the inheritance of the developmental loss of activity and for genetic-physiological studies on the role of AI activity in sucrose accumulation. Burger et al. (2002) studied the inheritance of sucrose accumulation between the extreme low-sucrose genotype FAQ and the high-sucrose genotype NY, and reported that a major gene may determine the trait of sucrose accumulation. Other studies have generally indicated that sugar levels are a quantitative genetic trait (Sinclair et al., 2006). The difference may be explained by the choice of genetic material used. The FAQ genotype may be extreme in its lack of sucrose accumulation, thereby allowing the identification of a major gene controlling the trait, whereas other low-sugar genotypes studied may be intermediate in their sucrose accumulation metabolism, allowing for the identification of quantitative trait loci with lesser, but significant, contributions.

In summary, three major conclusions have been made in this report. First, sucrose accumulation in melon genotypes is a function of the length of the sucrose accumulation period, which is primarily determined by the developmental loss of AI activity and the subsequent period until ripening and abscission. Second, threshold activities of the three enzymes SPS, SuSy, and NI also characterize sucrose-accumulating genotypes and they positively contribute to the sucrose accumulation period. Third, there is genetic variability in C. melo in determining the length of the accumulation period. Additional studies based on these genotypes are expected to shed further light on the important horticultural phenomenon of sucrose accumulation that determines fruit quality.

\section{Literature Cited}

Avigad, G. 1982. Sucrose and other disaccharides, p. 217-234. In: F.A. Loewus and W. Tannerw (eds.). Encyclopedia of plant physiology. Vol. 134. Springer Verlag, Heidelberg, Germany.

Burger, Y., S. Shen, M. Petreikov, and A.A. Schaffer. 2000. The contribution of sucrose to total sugar content in melons. Acta Hort. 510:479-485.

Burger, Y., Y. Yeselson, U. Saar, N. Katzir, I. Levin, and A.A. Schaffer. 2002. A single recessive gene for sucrose accumulation in Cucumis melo fruit. J. Amer. Soc. Hort. Sci. 127:938-943. 
Chen, J.K. and C.C. Black. 1992. Biochemical and immunological properties of alkaline invertase isolated from sprouting soybean hypocotyl. Arch. Biochem. Biophys. 295:61-69.

Chrost, B. and K. Schmitz. 1997. Changes in soluble sugars and activity of $\alpha$-galactosidases and acid invertase during muskmelon (Cucumis melo L.) fruit development. J. Plant Physiol. 151:41-50.

Gao, Z., M. Petreikov, E. Zamski, and A.A. Schaffer. 1999. Carbohydrate metabolism during early fruit development of sweet melon (Cucumis melo). Physiol. Plant. 106:1-8.

Giaquinta, R.T. 1979. Sucrose translocation and storage in the sugar beet. Plant Physiol. 63:828-832.

Glasziou, K.T. and K.R. Gayler. 1972. Storage of sugars in stalks of sugar cane. Bot. Rev. 38:471-488.

Goldschmidt, E.E. and S.C. Huber. 1992. Regulation of photosynthesis by end-product accumulation in leaves of plants storing starch, sucrose and hexose sugars. Plant Physiol. 99:1443-1448.

Hatch, M.D. and K.T. Glasziou. 1963. Sugar accumulation in sugarcane. II. Relationship of invertase activity of sugar content and growth rate in storage tissue of plants grown in controlled environments. Plant Physiol. 38:344-388.

Helmerhorst, E. and G.B. Stokes. 1980. Microcentrifuge desalting: A rapid, quantitative method for desalting small amounts of protein. Anal. Biochem. 104:130-135.

Hubbard, N.L., S.C. Huber, and D.M. Pharr. 1989. Sucrose phosphate synthase and acid invertase as determinates of sucrose concentration in developing muskmelon (Cucumis melo L.) fruits. J. Amer. Soc. Hort. Sci. 91:1527-1534.

Hubbard, N.L., S.C. Huber, and D.M. Pharr. 1990. Sucrose metabolism in ripening fruits as affected by leaf area. J. Amer. Soc. Hort. Sci. 115:798-802.

Hubbard, N.L., D.M. Pharr, and S.C. Huber. 1991. Sucrose phosphate synthase and other sucrose metabolism enzymes in fruit of various species. Physiol. Plant. 82:191-196.

Hughes, D.L. and M. Yamaguchi. 1983. Identification and distribution of some carbohydrates of the muskmelon plant. HortScience 18:739740.

Irving, D.E., P.L. Hurst, and J.S. Ragg. 1997. Changes in carbohydrates and carbohydrate metabolizing enzymes during the development, maturation, and ripening of buttercup squash (Cucurbita maxima D. 'Delica'). J. Amer. Soc. Hort. Sci. 122:310-314.

Iwatsubo, T., H. Nakagawa, N. Ogura, T. Hirabyashi, and T. Sato. 1992. Acid invertase of melon fruits: Immunochemical detection of acid invertases. Plant Cell Physiol. 33:1127-1133.

Kato, T. and S. Kubota. 1978. Properties of invertase in sugar storage of citrus fruit and changes in their activities during maturation. Physiol. Plant. 42:67-72.

Klann, E.M., B. Hall, and A.B. Bennett. 1993. Expression of acid invertase gene controls sugar composition in tomato (Lycopersicon) fruit. Plant Physiol. 103:863-870.

Klann, E.M., B. Hall, and A.B. Bennett. 1996. Antisense acid unvertase (TIV1) gene alters soluble sugar composition and size in transgenic fruit. Plant Physiol. 112:1321-1330.

Langenkamper, G., R. McHale, R.C. Gardner, and E. MacRae. 1998. Sucrose-phosphate synthase steady-state mRNA increases in ripening kiwifruit. Plant Mol. Biol. 36:857-869.

Laporte, M.M., J.A. Galagan, J.A. Shapiro, M.R. Boersig, C.K. Shewmaker, and T.D. Sharkey. 1997. Sucrose phosphate synthase activity and yield analysis of tomato plants transformed with maize sucrose-phosphate synthase. Planta 203:253-259.

Lester, G.E., L.S. Arias, and M. Gomez-Lim. 2001. Muskmelon fruit soluble acid invertase and sucrose phosphate activity and polypeptide profiles during growth and maturation. J. Amer. Soc. Hort. Sci. 126:33-36.

Lingle, S. and J.R. Dunlap. 1987. Sucrose metabolism in netted muskmelon fruit during development. Plant Physiol. 84:386-389.

McCollum, T.G., D.J. Huber, and D.J. Cantliffe. 1988. Soluble sugar accumulation and activity of related enzymes during muskmelon fruit development. J. Amer. Soc. Hort. Sci. 113:399-403.
Miron, D., M. Petreikov, N. Carmi, S. Shen, I. Levin, D. Granot, E. Zamski, and A.A. Schaffer. 2001. Invertase localization, gene expression, and sucrose uptake in developing fruit of L. esculentum and in the sucrose-accumulating L. hirsutum. Physiol. Plant. 115: 35-47.

Miron, D. and A.A. Schaffer. 1991. Sucrose phosphate synthase, sucrose synthase and invertase activities in developing fruit of Lycopersicon esculentum Mill. and the sucrose accumulating Lycopersicon hirsutum Humb. and Bonpl. Plant Physiol. 95:623627.

Mitchell, D.E., M.V. Gadus, and M.A. Madore. 1992. Patterns of assimilate production and translocation in muskmelon (Cucumis melo L.) I. Diurnal patterns. Plant Physiol. 99:959-996.

Moriguchi, T., K. Abe, T. Sanada, and S. Yamaki. 1992. Levels and role of sucrose synthase, sucrose phosphate synthase and acid invertase in sucrose accumulation in fruit of Asian pear. J. Amer. Soc. Hort. Sci. 117:274-278.

Moriguchi, T., T. Sanda, and S. Yamaki. 1990. Seasonal fluctuation of some enzyme relating to sucrose and sorbitol metabolism in peach fruit. J. Amer. Soc. Hort. Sci. 115:278-281.

Nguyen-Quoc, B., H. N'Tchobo, C.H. Foyer, and S. Yelle. 1999. Overexpression of sucrose phosphate synthase increases sucrose unloading in transformed tomato fruit. J. Expt. Bot. 50:785-791.

Paris, H.S., H. Nerson, and Z. Karchi. 1986. Branching of birdsnesttype melons. Crop Res. 26:33-40.

Pharr, D.M. and N.T. Hubbard. 1994. Melons: Biochemical and physiological control of sugar accumulation, p. 25-37. In: C.J. Arntzen (ed.). Encyclopedia of agricultural science. Vol. 3. Academic Press, New York.

Pitrat, M., P. Hanelt, and K. Hammer. 2000. Some comments on infraspecific classification on cultivars of melon. Acta Hort. 510:2936.

Pratt, H.K. 1970. Melons, p. 207-232. In: A.C. Hulme (ed.). The biochemistry of fruit and their products. Vol. 2. Academic Press, New York

Quick, W.P. and A.A. Schaffer. 1996. Sucrose metabolism in sources and sinks, p. 115-156. In: E. Zamski, and A.A. Schaffer (eds.). Photoassimilate distribution in plants and crops. Marcel Dekker, New York.

Ranwala, A.P., S.S. Iwanami, and H. Masuda. 1991. Acid and neutral invertases in the mesocarp of developing muskmelon (Cucumis melo L. cv Prince) fruit. Plant Physiol. 96:881-886.

Ricardo, C.P.P. and T.A.P. Rees. 1970. Invertase activity during the development of carrot roots. Phytochemistry 9:239-247.

Rosa, J.T. 1928. Change in composition during ripening and storage of melons. Hilgardia 3:421-443.

Sacher, J.A., M.D. Hatch, and A.T. Glasziou. 1963. Sugar accumulation cycle in sugar cane. III. Physical and metabolic aspects of cycle in immature storage tissues. Plant Physiol. 38:348-354.

Schaffer, A.A., B. Aloni, and E. Fogelman. 1987. Sucrose metabolism and accumulation in developing fruit of Cucumis. Phytochemistry 26:1883-1887.

Schaffer, A.A., Y. Burger, Z. Zheng, Z. Gao, D. Granot, M. Petreikov, Y. Yeselson, and S. Shen. 2000. Biochemistry of sugar metabolism in melons as related to the genetic improvement of fruit quality. Acta Hort. 510:449-453.

Schaffer, A.A., D.M. Pharr, and M.A. Madore. 1996. Cucutbits, p. 729-757. In: E. Zamski and A.A. Schaffer (eds.). Photoassimilate distribution in plants and crops. Marcel Dekker, New York.

Schaffer, A.A., I. Rylski, and M. Fogelman. 1989. Carbohydrate content and sucrose metabolism in developing Solanum muricatum fruits. Phytochemistry 28:737-739.

Sinclair, J.W., S.O. Park, G.E. Lester, K.S. Yoo, and K.M. Crosby. 2006. Identification and confirmation of RAPD markers and andromonoecious associated with quantitative trait loci for sugars in melon. J. Amer. Soc. Hort. Sci. 131:360-371.

Stepansky, A., I. Kovalski, A.A. Schaffer, and R. Perl-Ttreves. 1999. Variation in sugar levels and invertase activity in mature fruit 
representing a broad spectrum of Cucumis melo genotypes. Genet. Resour. Crop Evol. 45:53-62.

Stommel, J.R. 1992. Enzymatic components of sucrose accumulation in the wild tomato species Lycopersicon peruvianum. Plant Physiol. 99:324-328.

Sumner, J.B. 1921. Dinitro-salicilic acid, a reagent for estimation of sugars in normal and diabetic urine. J Biol. Chem. 47:5-7.

Suzuki, A., Y. Kanayama, and S. Yamaki. 1996. Occurrence of two sucrose synthase isozymes during maturation of japanese pear fruit. J. Amer. Soc. Hort. Sci. 121:943-947.

Van Handel, E. 1968. Direct microdetermination of sucrose. Anal. Biochem. 22:280-283.
Welles, G.W.H. and K. Buitlaar. 1988. Factors affecting soluble solids content in melons. Netherlands J. Agr. Sci. 36:239-246.

Yamaguchi, M., D.L. Hughes, K. Yabumoto, and W.C. Jennings. 1977. Quality of cantaloupes: Variability and attributes. Sci. Hort. 6:59-70.

Yelle, S., R.T. Chetelat, M. Dorais, J.W. De Verna, and A.B. Bennett. 1991. Sink metabolism in tomato fruit. IV. Genetic and biochemical analysis of sucrose accumulation. Plant Physiol. 95:1026-1035.

Zhu, Y.J., E. Komor, and P.H. Moore. 1997. Sucrose accumulation in the sugarcane stem is regulated by the difference between the activities of soluble acid invertase and sucrose phosphate synthase. Plant Physiol. 115:609-616. 